\title{
Functional Studies of the T295M Mutation Causing Glut1 Deficiency: Glucose Efflux Preferentially Affected by T295M
}

\author{
DONG WANG, HONG YANG, LEI SHI, LI MA, TATSUYA FUJII, KRISTIN ENGELSTAD, JUAN M. PASCUAL, AND
} DARRYL C. DE VIVO

\begin{abstract}
Department of Neurology [D.W., H.Y., K.E., D.C.V.], Department of Physiology and Cellular Biophysics [L.M.], Columbia University, New York, New York 10032; Department of Physiology and Biophysics [L.S.], Cornell University, New York, New York 10021; Department of Pediatrics [T.F.], Shiga Medical Center for Children, Moriyama, 524-0022, Japan; Department of Neurology [J.M.P.], The University of Texas Southwestern Medical Center, Dallas, Texas 75390
\end{abstract}

\begin{abstract}
Glucose transporter type 1 (Glut1) deficiency syndrome (Glut1 DS, OMIM: \#606777) is characterized by infantile seizures, acquired microcephaly, developmental delay, hypoglycorrhachia (CSF glucose $<40 \mathrm{mg} / \mathrm{dL}$ ), and decreased erythrocyte glucose uptake (56.1 $\pm 17 \%$ of control). Previously, we reported two patients with a mild Glut 1 deficiency phenotype associated with a heterozygous GLUT1 T295M mutation and normal erythrocyte glucose uptake. We assessed the pathogenicity of T295M in the Xenopus laevis oocyte expression system. Under zero-trans influx conditions, the $\mathrm{T} 295 \mathrm{M} \mathrm{V}_{\max }(590 \mathrm{pmol} / \mathrm{min} /$ oocyte $)$ was $79 \%$ of the WT value and the $\mathrm{Km}(14.3 \mathrm{mM})$ was increased compared with WT $(9.6 \mathrm{mM})$. Under zero-trans efflux conditions, both the $\mathrm{V}_{\max }(1216 \mathrm{pmol} / \mathrm{min} /$ oocyte) and $\mathrm{Km}(8.8 \mathrm{mM})$ in $\mathrm{T} 295 \mathrm{M}$ mutant Glut1 were markedly decreased in comparison to the WT values (7443 pmol/min/oocyte and $90.8 \mathrm{mM}$ ). Western blot analysis and confocal studies confirmed incorporation of the T295M mutant protein into the plasma membrane. The side chain of M295 is predicted to block the extracellular "gate" for glucose efflux in our Glut-1 molecular model. We conclude that the T295M mutation specifically alters Glut1 conformation and asymmetrically affects glucose flux across the cell by perturbing efflux more than influx. These findings explain the seemingly paradoxical findings of Glut1 DS with hypoglycorrhachia and "normal" erythrocyte glucose uptake. (Pediatr Res 64: 538-543, 2008)
\end{abstract}

$T^{T}$ he facilitative glucose transporter type 1 (Glut1) was the first identified member of the glucose transporter protein family (GLUT), which now has 13 members (Glut1-12 and HMT1) (1). Glut1 has two distinct molecular forms with apparent molecular weights of 55 and $45 \mathrm{kD}$. These two forms are encoded by the same gene and differ only by the extent of glycosylation (2). Glucose needs to traverse the endothelial cell luminal membrane, cytoplasm, and abluminal membrane to enter the brain extracellular pool. Glut1 is distributed asymmetrically on the luminal and abluminal endothelial membranes as shown by immunogold electron microscopy and by Cytochalasin B binding in human, rabbit, rat, squirrel, and monkey (3). Other studies suggest a more symmetrical distribution of Glut1 between the luminal and abluminal membranes (4). GLUT1 expression is regulated by metabolic

Received March 25, 2008; accepted June 20, 2008.

Correspondence: Darryl C. De Vivo, M.D., Columbia University, Neurological Institute, 710 West 168th Street, New York, NY, 10032; e-mail: dcd1@columbia.edu

Supported by NS37949 and 1 UL1 RR024156-01(DCD), the Will and the Colleen Giblin Foundations (DCD), K12-NS01698 (DW, JMP), and RPB, Inc. demand and regional glucose metabolic rate. The cytoplasmic Glut1 can modulate glucose transport by translocation into the luminal membrane. The ratio of luminal to abluminal Glut1 can have a directive effect on rates of glucose uptake into brain (5).

The functional roles of single amino acids have been studied in some detail. Mutations have been directed at amino acid residues that are highly conserved or apparently critical for catalytic activity (reviewed by McGowan KM et al. 1995; Hruz PW and Mueckler MM 2001) (6,7). The mutants Q282L (8), P385I (9), and truncation of the last 27 carboxyl terminal residues (10) selectively reduce $2-\mathrm{N}-4$-(1-azi-2,2,2-trifluoroethyl) benzoyl-1,3-bis(D-mannos-4-yloxy)-2-propylamine (ATB-BMPA) labeling. The mutants Y293I (11), E329Q, E393D, and R400L (12) selectively reduce Cytochalasin B binding. In contrast, the mutants W412L (13-15), R $92 \mathrm{~L}$, and R333L/R334/A (16) reduce the glucose transport activity with very little effect on ATB-BMPA photolabeling and Cytochalasin B binding. However, E146D and R153L mutants markedly reduce transport activity, ATB-BMPA photolabeling and Cytochalasin B binding (12). Also, whole transmembrane domains or other conserved domains have been studied by site-directed mutagenesis $(7,17)$ providing additional insights into the structure/function relationships within Glut1.

Glut1 Deficiency Syndrome (Glut1 DS) was first described by De Vivo et al. in 1991 (18). About 200 patients have been diagnosed worldwide since the first report. Glut1 DS is caused by impaired facilitative glucose transport across the BBB. Fluorescence in situ hybridization and mutational analysis of the GLUT1 gene has revealed either hemizygosity or heterozygosity for missense, nonsense, insertion, deletion, duplication, and splice site mutations (19).

We previously reported two Glut1 DS patients $(20,21)$ who had very similar clinical findings, the same GLUT1 missense mutation (T295M), and a normal 3-O-methyl-D-glucose (3OMG) uptake under zero-trans influx conditions (102\% and $114 \%$ of control values) in erythrocytes. All other Glut1 DS patients studied by us thus far (116 cases) have had decreased erythrocyte glucose uptake. This report explains this apparent paradox by studying the pathogenicity of this T295M missense mutation in the Xenopus laevis oocyte expression system. Our experimental findings document that the T295M mutation disturbs Glut1 efflux activity preferentially. These findings harmonize the seemingly paradoxical clinical obser- 
vation of hypoglycorrhachia and "normal" erythrocyte glucose uptake.

\section{METHODS}

The investigatory studies on blood were exempted from full review and approved by the Columbia University Institutional Review Board. Informed consent was obtained from the parents and patients who were participants in this study.

3-OMG uptake into freshly isolated erythrocytes were performed as previously described (22).

Mutational analysis of the GLUT1 gene isolated from white blood cells was done as previously described (19).

Mutagenesis and cRNA preparation was from cDNA templates by using pM-GLUT1 plasmid DNA (23) as the template for T295 mutant construction. T295M was created by using the Quick Change Site-Directed Mutagenesis Kit (Cat \#200519, Stratagene, La Jolla, CA 92037) with the following primers (F, GTCTTCTATTACTCCATGAGCATCTTCGAGAAGGCG, R, CGCCTTCTCGAAGATGCTCATGGAGTAATAGAAGAC). The presence of the T295M mutation was confirmed by DNA sequence analysis. Capped, runoff cRNA transcripts of WT and mutant GLUT1 were synthesized from pM-GLUT1 constructs after linearization at a unique Not I site using a mMessage mMachine kit (Ambion, Austin, TX). cRNAs were quantified by incorporation of ${ }^{3} \mathrm{H}$-UTP tracer in the transcription reaction and their integrity verified by denaturing agarose gel electrophoresis. cRNAs were stored at $-80^{\circ} \mathrm{C}$ until the time of dilution in $100 \mathrm{mM} \mathrm{KCl}$ for injection.

Xenopus oocytes were prepared as previously described (23). The largest undamaged oocytes (stages V and VI) were transferred to Barth's solution and incubated overnight at $18^{\circ} \mathrm{C}$ and injected the following day. Oocytes were injected with either $50 \mathrm{~nL}$ of water, $50 \mathrm{~nL}$ of wild-type cRNA, or $50 \mathrm{~nL}$ of T295M mutant cRNA (1 ng/nL) and were kept in Barth's medium at $18^{\circ} \mathrm{C}$ for $72 \mathrm{~h}$

Zero-trans influx studies of 3-OMG into Xenopus oocytes were determined as described previously (23). Uptake of ${ }^{14} \mathrm{C}$ labeled 3-OMG together with unlabeled 3-OMG $(0-50 \mathrm{mM})$ was determined from groups of 5 oocytes under zero-trans influx. Uptake values from oocytes injected with water were subtracted from all groups.

Zero-trans efflux studies of 3-OMG into Xenopus oocytes was performed as previously described (23). Briefly, Zero-trans efflux of 3-OMG into Xenopus Oocytes was performed by injecting different amounts of ${ }^{14} \mathrm{C}$ labeled 3-OMG together with unlabeled 3-OMG to reach calculated concentrations of 1 to $90 \mathrm{mM}$ in oocytes expressing wild-type and mutant T295M Glut1 (assuming a $0.5 \mathrm{~nL}$ water volume in an average oocyte) (24). After injection, immediately before the efflux assay, oocytes were quickly washed three times in $2 \mathrm{~mL}$ of Barth's solution and transferred to a scintillation vial containing $0.5 \mathrm{~mL}$ of Barth's solution. After 2 minutes, the oocytes were transferred to another scintillation vial. About $0.5 \mathrm{~mL} 0.1 \%$ SDS was added to both vials and mixed by vortex. Five microliters Hionic-Fluor scintillation fluid (Packard Bioscience B. V., Groningen, the Netherlands) were added before counting. The measured amount of glucose in both vials was used to evaluate the actual intracellular glucose concentration during the efflux measurement. The efflux rate from control oocytes (oocytes injected with water instead of cRNA) was subtracted when calculating both the wild type and T295M mutant expressing oocytes.

Confocal immunofluorescence microscopy for detecting Glut1 in frozen Xenopus laevis oocyte sections was performed as described previously (23). Goat polyclonal IgG (Glut1 C20) (Santa Cruz Biotechnology, Santa Cruz, CA) was used as primary antibody, and Alexa Fluor 568 conjugated donkey anti-goat IgG (Molecular Probes, Eugene, OR) was used as the secondary antibody. Confocal microscopy was performed at the Optical Microscopy Facility at Columbia University.

Western blot analysis of membranes from injected oocytes (the same batch of oocytes used for uptake studies) were prepared by a modified method (23). All fractions were stored at $-80^{\circ} \mathrm{C}$ until use. Western blot analysis was performed as described previously (25). Briefly, $15 \mu \mathrm{L}$ of total membrane or purified plasma membrane samples from wild-type, mutant, and $\mathrm{H}_{2} \mathrm{O}$ injected oocytes were subjected to 4 to $20 \%$ SDS-polyacrylamide gradient gel electrophoresis. Rabbit-anti-Glut-1 antibody (FabGennic Inc, Frisco, TX) was used as primary antibody, and donkey anti-rabbit IgG-HRP (Santa Cruz Biotechnology, Santa Cruz, CA) was used as the secondary antibody. Glut1 signals were quantified digitally using a densitometer equipped with ImageQuant software (Molecular Dynamics, CA). The densities of wild-type Glut1 signals in purified oocyte membranes were used to normalize mutant Glut1 densities.
Transport kinetics was analyzed by fitting data points to the MichaelisMenten equation utilizing Prism 4 software (OriginLab Software, Inc., Northampton, MA).

A human Glut1 molecular model was constructed with the homology modeling algorithm Modeler 8v2 based on the crystal structures of LacY (PDB code: 2CFQ) (26) and GlpT (PDB code: 1PW4) (27). The sequence alignment used in the modeling was based on a) sequence-structure alignments of the human Glut1 sequence and the LacY and GlpT structures with FUGUE (28) and b) the pair wise structural alignment of LacY and GlpT calculated by Structal (29) excluding the $\mathrm{N}$ - and C-termini, which diverge significantly. The initial sequence alignment was further refined and validated by comparing the accessible residue positions detected so far in Glut1 by the substituted cysteine accessibility method (30) by Mueckler et al. (31) with the solvent accessible surface calculated by InsightII (Accelrys, San Diego, CA) of both LacY and GlpT structures. Based on the WT model, the T295M mutant was constructed with Pymol (DeLano Scientific, Palo Alto, CA).

\section{RESULTS}

Patient 1. A 16-year-old boy was born after an uneventful pregnancy and term delivery. His birth weight was $7 \mathrm{lbs} 12$ ounces and the Apgar scores were 9 and 10. At age $1 \mathrm{y}$, mother noticed isolated myoclonic jerks of the body, later increasing into clusters by 18 mo. By age $4 \mathrm{y}$, the jerks became increasingly evident upon awakening during which he also appeared anarthric and dazed. The symptoms improved after taking some sugar-containing foods. At age three and half years he also developed an acute "gait disorder." The disturbance included writhing movements of the limbs, body and neck, and gyrations of the pelvis with a sudden onset lasting minutes to an hour. The movements could be interrupted by feeding sugar-containing foods. His early motor development was normal. He sat at 6 mo, walked with assistance at $13 \mathrm{mo}$, and walked independently at $21 \mathrm{mo}$. He had poor coordination and delayed speech and language development. At age $3 \mathrm{y}$, he was treated with phenytoin and phenobarbital for myoclonic seizures. Blood studies and head CT scan were normal. Electroencephalography (EEG) revealed bursts of $3 \mathrm{~Hz}$ spike and wave discharges correlating with myoclonic jerks. At age $5 \mathrm{y}$, he was tried on other antiepileptic drugs without clear benefit and the brain MRI scan was normal. At age $8 \mathrm{y}$, a lumber puncture showed a low CSF glucose $(37.8 \mathrm{mg} / \mathrm{dL})$, low normal lactate $(0.97 \mathrm{mM})$, and normal blood glucose $(94 \mathrm{mg} / \mathrm{dL})$. The erythrocyte 3-OMG uptake was normal. Glut-1 DS was diagnosed and a ketogenic diet was started. Little or no benefit was reported because of his noncompliance with the diet regimen. At age $10 \mathrm{y}$, the ketogenic diet was switched to a high sugar diet with cornstarch supplementation and a frequent (q2h) meals/snacks plan. He still had myoclonic jerks, worse in the morning, occasional unsteady gait, writhing movements of head and upper extremities and disturbed mood characterized by being mute and nonresponsive. The ketogenic diet was restarted at age $15 \mathrm{y}$. He improved socially and academically and was generally asymptomatic during day. He still had "wobbly legs" after walking a distance (2-5 min walk).

Patient 2. A 10-year-old girl was born to healthy nonconsanguineous parents after an uneventful pregnancy and term delivery with normal birth weight and head circumference. Her motor milestones in infancy were normal and she walked at age 15 mo. Her head growth decelerated after age 5 mo when she first developed a generalized tonic-clonic convul- 
sion, and the head circumference fell below the third percentile after age $2 \mathrm{y}$. EEG at age 8 mo was normal. She had seizures once a month, mostly in the morning, and an EEG at age $1 \mathrm{yr}$ showed spike discharges bilaterally over the central areas. Carbamazepine was ineffective, but zonisamide decreased the seizure frequency. After age $2 \mathrm{y}$, convulsions were followed by nausea and vomiting for about $6 \mathrm{~h}$. She had akinetic (generalized paralytic) seizures with retained consciousness. She also had "fits of unsteady gait" for a few hours. This intermittent ataxic gait improved after taking a nap or having a meal. At $5 \mathrm{y}$, she had mild truncal ataxia and slurred speech. She could speak in sentences. Patellar tendon reflexes were increased and plantar reflexes were extensor. Funduscopy was normal. Brain MRI and EEG were normal. Serum levels of alpha-fetoprotein, electrolytes, lactate, pyruvate, and urinary organic acids were normal. The CSF lactate and pyruvate levels were 1.0 and $0.06 \mathrm{mM}$. The CSF glucose level was only $31 \mathrm{mg} / \mathrm{dL}$ whereas the blood glucose was 77 $\mathrm{mg} / \mathrm{dL}$. Ketogenic diet (1.5:1 ratio) was started at age 107 mo. Her truncal ataxia significantly improved within a week of initiating the ketogenic diet, but she still had convulsions, akinetic seizures or transient alternating hemiplegia followed by vomiting. After a 2:1 ketogenic diet was started, convulsions disappeared and akinetic seizures occurred less frequently. The two patients' clinical features, laboratory data, and erythrocyte 3-OMG uptake (zero-trans influx) kinetics are summarized in Tables 1 and 2.

Mutagenesis studies in xenopus oocytes. The Thr hydrophilic side chain was replaced by a larger Met hydrophobic side chain in T295M. The kinetic parameters and curves of 3-OMG uptake under zero-trans influx and efflux conditions are shown in Fig. $1 A$ and $1 B$. Under zero-trans influx conditions, the $\mathrm{Km}$ and $\mathrm{V}_{\max }$ of $\mathrm{T} 295 \mathrm{M}$ mutant Glut1 were 14.3 $\mathrm{mM}$ and $590 \mathrm{pmol} / \mathrm{min} /$ oocyte. The $\mathrm{Km}$ and $\mathrm{V}_{\max }$ of WT Glut1 were $9.6 \mathrm{mM}$ and $747 \mathrm{pmol} / \mathrm{min} /$ oocyte. Under zerotrans efflux conditions, the $\mathrm{Km}$ and $\mathrm{V}_{\max }$ of $\mathrm{T} 295 \mathrm{M}$ mutant were $8.8 \mathrm{mM}$ and $1216 \mathrm{pmol} / \mathrm{min} /$ oocyte, the $\mathrm{Km}$ and $\mathrm{V}_{\max }$ of WT Glut1 were $90.8 \mathrm{mM}$ and $7443 \mathrm{pmol} / \mathrm{min} /$ oocyte.

To document targeting of the mutant Glut1 transporters to the plasma membrane, confocal microscopy of Xenopus oo-


Figure 1. Oocytes injected with $50 \mathrm{ng}$ cRNA encoding WT and T295M Glut1, or $50 \mathrm{~nL}$ water were used 3 days later for the assay. Uptake values from oocytes injected with water were subtracted from all groups. Values represent the mean \pm Standard error. The data were fitted to the Michaelis-Menten equation using Prism 4 software $\square$ : WT, $\mathbf{\Delta}$ : T295M. A, Zero-trans influx of 3-OMG was performed with 5 oocytes in Bath's medium containing 3-OMG $(0-50 \mathrm{mM})$. B, Zero-trans efflux of 3-OMG was performed with oocytes injected with ${ }^{14} \mathrm{C}$ labeled 3-OMG together with unlabeled 3-OMG to reach calculated concentrations of 1 to $90 \mathrm{mM}$.

cytes expressing WT and T295M mutant Glut1 was performed. T295M mutant Glut-1 was targeted to the plasma membrane similar to the WT Glut-1 (Fig. 2A). Western blot analysis of the purified plasma membrane and, the relative amounts of T295M mutant Glut1 compared with the wild-type Glut1 immunoreactivity in plasma membrane are shown in Fig. $2 B$. The $\mathrm{V}_{\max }$ values of mutants (Fig. $1 A$ and $1 B$ ) nor-

Table 1. Clinical features of the two patients

\begin{tabular}{|c|c|c|c|c|c|c|c|c|c|}
\hline Patient & Age (y) & Sex & $\begin{array}{l}\text { Seizure } \\
\text { onset* }\end{array}$ & $\begin{array}{l}\text { Seizures } \\
\text { frequency }\end{array}$ & $\begin{array}{c}\text { Hypotonia/ } \\
\text { spasticity }\end{array}$ & Ataxia & Language deficit & $\mathrm{HC} \ddagger$ & $\mathrm{KD} \dagger$ \\
\hline 1 & 12 & M & 12 & $1 / \mathrm{mo}$ & Hypotonia & $\begin{array}{l}\text { Ataxia, able } \\
\text { to walk }\end{array}$ & $\begin{array}{l}\text { Speaks in sentence } \\
\text { decreased vocabulary }\end{array}$ & 3 & 96 \\
\hline $2 \S$ & 10 & $\mathrm{~F}$ & 5 & $1 / \mathrm{mo}$ & Spasticity & Truncal ataxia & $\begin{array}{l}\text { Speaks in sentence, } \\
\text { slurred speech }\end{array}$ & $<3$ & 107 \\
\hline
\end{tabular}

\footnotetext{
* Seizure onset age in months. $\ddagger$ HC-head circumference in percentile. $\dagger$ KD: Ketogenic diet started age in months. § data published previously (21).
}

Table 2. Lumbar puncture data and 3-OMG erythrocyte uptake (zero-trans influx)

\begin{tabular}{|c|c|c|c|c|c|c|c|c|}
\hline \multirow[b]{2}{*}{ Patient } & \multirow{2}{*}{$\begin{array}{c}\text { CSF } \\
\text { glucose }\end{array}$} & \multirow{2}{*}{$\begin{array}{l}\text { Blood } \\
\text { glucose }\end{array}$} & \multirow{2}{*}{$\begin{array}{c}\mathrm{CSF} \\
\text { lactate }\end{array}$} & \multirow{2}{*}{$\begin{array}{l}\text { 3-OMG } \\
\text { uptake }\end{array}$} & \multicolumn{2}{|c|}{$V \max$} & \multicolumn{2}{|c|}{$\mathrm{Km}$} \\
\hline & & & & & Patient & Control & Patient & Control \\
\hline 1 & 38 & 94 & 1.0 & $102 \%$ & 2500 & 3333 & 2.3 & 2.0 \\
\hline $2 *$ & 31 & 77 & 1.0 & $114 \%$ & 2500 & 2667 & 2 & 1.75 \\
\hline
\end{tabular}

Glucose values in mg/dL. CSF lactate values in mM. Vmax: fmol/s/10 RBC. Km: mM.

* Data published previously (21). 


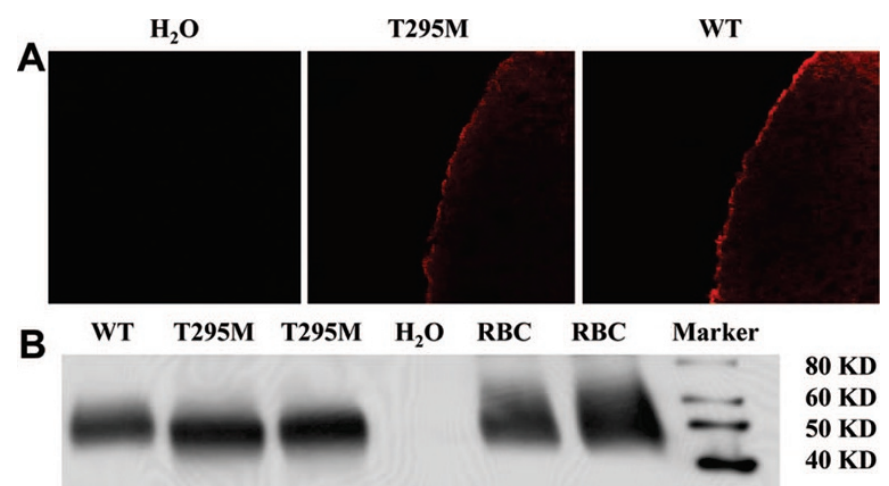

Figure 2. Glut1 expression in Xenopus oocytes. A, Localization of mutant Glut1 in oocytes by confocal immmunofluorescence microscopy. $B$, Western blot analysis of purified plasma membrane from Xenopus oocytes. The averaged densities of WT Glut1 signals in purified oocyte membranes were used to normalize T295M mutant Glut1 averaged densities. Red blood cell membrane protein was loaded as a positive control. The size of molecular weight marker is indicated on the right.

malized by the relative amount of Glut1 in purified plasma membrane were compared with the WT Glut1 to estimate the relative transport activity. The $\mathrm{Km}$ value of the T295M mutant protein $(14.3 \mathrm{mM})$ under zero-trans influx condition is higher than that of the WT Glut1 protein $(9.6 \mathrm{mM})$. The normalized $\mathrm{T} 295 \mathrm{M} \mathrm{V}_{\max }(590 \mathrm{pmol} / \mathrm{min} /$ oocyte $)$ is about $79 \%$ that of the WT value (747 $\mathrm{pmol} / \mathrm{min} /$ oocyte). Under conditions of zerotrans efflux, the $\mathrm{Km}$ value of the mutant T295M Glut1 (8.8 $\mathrm{mM})$ is much lower than the WT value $(90.8 \mathrm{mM})$. In addition, the $\mathrm{V}_{\max }$ value of the $\mathrm{T} 295 \mathrm{M}(1216 \mathrm{pmol} / \mathrm{min} /$ oocyte $)$ is only $16 \%$ of the WT value (7443 pmol/min/oocyte).

\section{DISCUSSION}

Glut1 DS (OMIM \#606777) is a disorder of brain energy metabolism caused by Glut1 haploinsufficiency (18). Since 1991, several hundred patients have been identified in the United States and elsewhere throughout the world (32-34). The laboratory signature of Glut1DS is hypoglycorrhachia and low CSF lactate concentration in the absence of hypoglycemia. All except three patients $(20,21)$ have shown decreased erythrocyte 3-OMG uptake (zero-trans influx) values, generally about $50 \%$ of control values. Two of these three exceptional patients shared a similar mild phenotype including monthly seizures beginning in infancy, developmental delay, ataxia, microcephaly, language deficit, hypoglycorrhachia, and low normal CSF lactate values (Tables 1 and 2). The erythrocyte zero-trans influx of 3-OMG was normal (Table 2) and both patients carried the heterozygous T295M mutation in GLUT1.

How could the normal 3-OMG uptake (under zero-transinflux conditions) in these two patients with typical clinical and biochemical features of Glut1 DS be explained? We hypothesized that $\mathrm{T} 295 \mathrm{M}$ caused little or no disturbance with influx, as seen in the erythrocyte glucose uptake assay, and more disturbance with efflux as reflected by the hypoglycorrachia. Glucose would diffuse across the luminal endothelial membrane (influx), enter the endothelial cytoplasmic compartment, and diffuse more slowly across the abluminal membrane
A

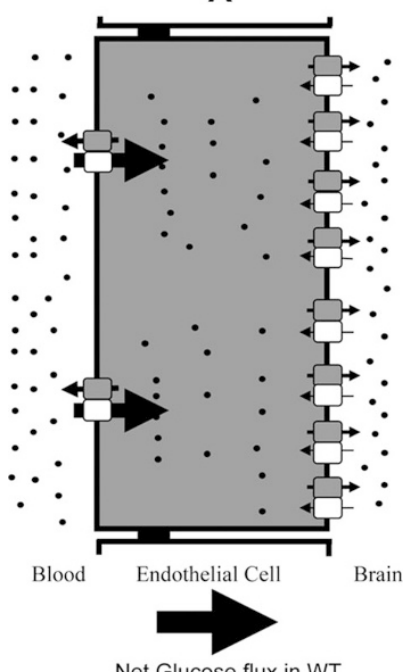

Net Glucose flux in WT

$\square \rightarrow$ WT Glut1 ( influx conformation)

$\rightarrow$ T295M Glut 1 (influx conformation) - Tight junction

Figure 3. A schematic diagram of glucose transport across the BBB. Glut-1 is asymmetrically expressed on the luminal and abluminal membranes of the endothelial cells in the BBB (based on luminal: abluminal ratio 1:4). The predicated net glucose flux across the BBB in the patient with the T295M mutation $(B)$ is half of the WT $(A)$ glucose flux based on the absolute mutagenesis study results in oocyte expression system. The oocyte expression system is homozygous for the mutant or WT Glut1, and the patient is heterozygous (as shown in the diagram).

(efflux) to the brain extracellular space (Fig. 3). Our mutagenesis studies as presented in this report support this hypothesis.

Helices 7 and 8 are predicted to cooperate in the formation of a central pore in different models $(17,35)$. T295 is located in the STS polar motif of the exofacial loop between helix 7 and 8 and is conserved in the mammalian glucose transporters 1 to 4 (36). Substitutions at Y293 in this loop impair transport activity by reducing accessibility to the cytoplasmic substratebinding site, effectively locking the transporter into an outward facing conformation (11). The T295 amino acid residue is about $4.9 \AA$ away from one of the nine clusters spanning the entire hydrophilic channel as revealed by docking studies (37) and locates along the extracellular opening of the transport channel (35). Mutagenesis studies of the T295A, T295S, and T295G Glut-4 mutants (amino acid residue corresponding to that of Glut1 in COS-7 cells) showed that T295A abolishes the glucose transport activity and markedly reduces the Cytochalasin B binding without affecting the photolabeling with ATBBMPA. However, the T295S and T295G mutants have a normal transport activity and ATB-BMPA labeling with normal to marginally reduced Cytochalasin B binding. Cystinescanning mutagenesis of the T295C Glut1 mutant in the Xenopus oocyte system correlated with normal glucose uptake (38). These observations reveal that both steric and polar influences are required at position T295. The steric influence predominates as the T295A markedly affects transport function whereas the T295G, T295S, and T295C mutations are associated with normal transport activity $(36,38)$. Recently, a T295M mutagenesis study in CHO cells correlated with a 

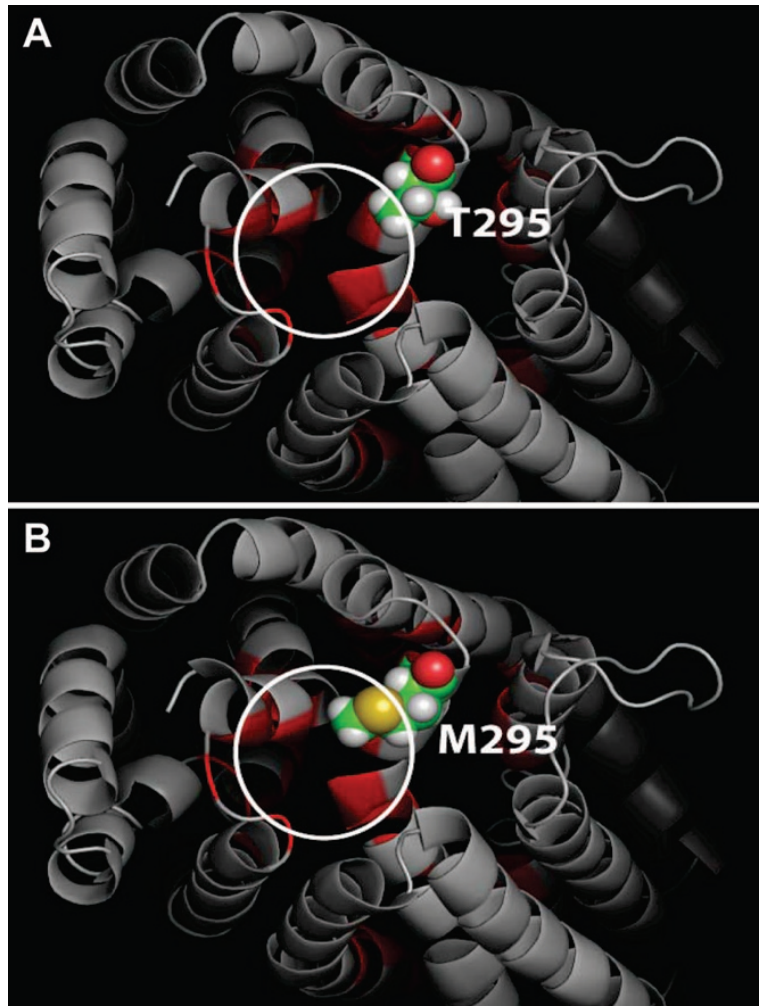

Figure 4. Glut1 model. The inward-facing Glut1 model is shown. TM helices are represented in gray. Glut1 residue 295 is strategically positioned at the "gate" (white circle). The side chains of residues Met295 and Thr295 are depicted. $A$, the WT Glut1 model from the extracellular aspect of the transporter. B, Glut-1 model with M295 side chain blocking the gate.

significant decrease in the 2-DOG uptake under zero-trans influx conditions (39). However, this finding does not explain the patients' normal 3-OMG erythrocyte uptake under zerotrans influx conditions. These data suggest that the exofacial loop between helices 7 and 8 plays an important role in the normal functional Glut-1 conformation maintenance.

Our mutagenesis studies in Хenopus oocytes support our hypothesis, namely, that the mutation exerts an asymmetric effect on the influx and efflux of glucose. The $\mathrm{V}_{\max }$ of the mutant T295M protein under 3-OMG zero-trans influx in oocytes is about $79 \%$ of control values (Fig. 1A). However, the $\mathrm{V}_{\max }$ of $\mathrm{T} 295 \mathrm{M}$ under zero-trans efflux is significantly decreased to about $16 \%$ of the WT values (Fig. $1 B$ ) and the T295M mutant Glut1 has a higher affinity $(\mathrm{Km} 8.8 \mathrm{mM})$ compared with the WT Glut-1 $(90.8 \mathrm{mM})$.

A recent Steered Molecular Dynamics study of LacY identified residues that may align along the substrate translocation pathway by pulling the substrate in a direction perpendicular to the membrane (40). In Figure 4, the Glut1 positions aligned to those pathway-forming residues of LacY are colored red. The LacY crystal structure is assumed to be in an inwardfacing conformation. Based on this crystal structure, our Glut-1 molecular model shows that the Glut1 residue 295 is strategically positioned at the "gate" (white circle) (Fig. 4). Blocking this gate with the larger side chain of Met should have a stronger impact on efflux. Influx needs an outwardfacing conformation and the narrower gate should be on the intracellular side of the transmembrane domain. Thus, the T295M does not have strong impact on influx.

In summary, the T295M mutation specifically alters the Glut1 conformation as indicated in the Glut1 molecular model, and significantly disrupts Glut 1 efflux activity while only mildly disrupting Glut1 influx activity. Under these abnormal conditions, glucose could be transported into the cells (such as erythrocytes, endothelial cells, and glial cells) efficiently, but would not be transported out of the cells efficiently (Fig. 3). This asymmetric kinetic defect could explain the laboratory results in these two patients. The kinetic abnormality would only be detected by erythrocyte glucose efflux studies, not influx studies as we do routinely. These findings also dictate that GLUT1 mutational analysis should be performed in the rare circumstances when the clinical and laboratory findings are consistent with the diagnosis of Glut1 DS but the erythrocyte glucose uptake (influx) studies are normal.

\section{REFERENCES}

1. Joost HG, Thorens B 2001 The extended GLUT-family of sugar/polyol transport facilitators: nomenclature, sequence characteristics, and potential function of its novel members (review). Mol Membr Biol 18:247-256

2. Birnbaum MJ, Haspel HC, Rosen OM 1986 Cloning and characterization of a cDNA encoding the rat brain glucose-transporter protein. Proc Natl Acad Sci USA $83: 5784-5788$

3. Pardridge WM 1991 Advances in cell biology of blood-brain barrier transport Semin Cell Biol 2:419-426

4. Simpson IA, Carruthers A, Vannucci SJ 2007 Supply and demand in cerebral energy metabolism: the role of nutrient transporters. J Cereb Blood Flow Metab 27:17661791

5. Simpson IA, Appel NM, Hokari M, Oki J, Holman GD, Maher F, Koehler-Stec EM, Vannucci SJ, Smith QR 1999 Blood-brain barrier glucose transporter: effects of hypo- and hyperglycemia revisited. J Neurochem 72:238-247

6. McGowan KM, Long SD, Pekala PH 1995 Glucose transporter gene expression: regulation of transcription and mRNA stability. Pharmacol Ther 66:465-505

7. Hruz PW, Mueckler MM 2001 Structural analysis of the GLUT1 facilitative glucose transporter (review). Mol Membr Biol 18:183-193

8. Hashiramoto M, Kadowaki T, Clark AE, Muraoka A, Momomura K, Sakura H, Tobe K, Akanuma Y, Yazaki Y, Holman GD, Kasuga M 1992 Site-directed mutagenesis of GLUT1 in helix 7 residue 282 results in perturbation of exofacial ligand binding. J Biol Chem 267:17502-17507

9. Tamori Y, Hashiramoto M, Clark AE, Mori H, Muraoka A, Kadowaki T, Holman GD, Kasuga M 1994 Substitution at Pro385 of GLUT1 perturbs the glucose transport function by reducing conformational flexibility. J Biol Chem 269:2982-2986

10. Muraoka A, Hashiramoto M, Clark AE, Edwards LC, Sakura H, Kadowaki T, Holman GD, Kasuga M 1995 Analysis of the structural features of the C-terminus of GLUT1 that are required for transport catalytic activity. Biochem J 311:699-704

11. Mori H, Hashiramoto M, Clark AE, Yang J, Muraoka A, Tamori Y, Kasuga M, Holman GD 1994 Substitution of tyrosine 293 of GLUT1 locks the transporter into an outward facing conformation. J Biol Chem 269:11578-11583

12. Schurmann A, Doege H, Ohnimus H, Monser V, Buchs A, Joost HG 1997 Role of conserved arginine and glutamate residues on the cytosolic surface of glucose transporters for transporter function. Biochemistry 36:12897-12902

13. Katagiri H, Asano T, Shibasaki Y, Lin JL, Tsukuda K, Ishihara H, Akanuma Y, Takaku F, Oka Y 1991 Substitution of leucine for tryptophan 412 does not abolish cytochalasin B labeling but markedly decreases the intrinsic activity of GLUT1 glucose transporter. J Biol Chem 266:7769-7773

14. Garcia JC, Strube M, Leingang K, Keller K, Mueckler MM 1992 Amino acid substitutions at tryptophan 388 and tryptophan 412 of the HepG2 (Glut1) glucose transporter inhibit transport activity and targeting to the plasma membrane in Xenopus oocytes. J Biol Chem 267:7770-7776

15. Schurmann A, Keller K, Monden I, Brown FM, Wandel S, Shanahan MF, Joost HG 1993 Glucose transport activity and photolabelling with 3-[125I]iodo-4azidophenethylamido-7-O-succinyldeacetyl (IAPS)-forskolin of two mutants at tryptophan-388 and -412 of the glucose transporter GLUT1: dissociation of the binding domains of forskolin and glucose. Biochem J 290:497-501

16. Wandel S, Schurmann A, Becker W, Summers SA, Shanahan MF, Joost HG 1995 Mutation of two conserved arginine residues in the glucose transporter GLUT4 supresses transport activity, but not glucose-inhibitable binding of inhibitory ligands. Naunyn Schmiedebergs Arch Pharmacol 353:36-41

17. Mueckler M, Makepeace C 2006 Transmembrane segment 12 of the Glut 1 glucose transporter is an outer helix and is not directly involved in the transport mechanism. J Biol Chem 281:36993-36998

18. De Vivo DC, Trifiletti RR, Jacobson RI, Ronen GM, Behmand RA, Harik SI 1991 Defective glucose transport across the blood-brain barrier as a cause of persistent hypoglycorrhachia, seizures, and developmental delay. N Engl J Med 325:703-709 
19. Wang D, Kranz-Eble P, De Vivo DC 2000 Mutational analysis of GLUT1 (SLC2A1) in Glut-1 deficiency syndrome. Hum Mutat 16:224-231

20. Wang D, Pascual JM, Yang H, Engelstad K, Jhung S, Sun RP, De Vivo DC 2005 Glut-1 deficiency syndrome: clinical, genetic, and therapeutic aspects. Ann Neurol 57:111-118

21. Fujii T, Ho YY, Wang D, De Vivo DC, Miyajima T, Wong HY, Tsang PT, Shirasaka Y, Kudo T, Ito M 2007 Three Japanese patients with glucose transporter type 1 deficiency syndrome. Brain Dev 29:92-97

22. Klepper J, Garcia-Alvarez M, O'Driscoll KR, Parides MK, Wang D, Ho YY, De Vivo DC 1999 Erythrocyte 3-O-methyl-D-glucose uptake assay for diagnosis of glucose-transporter-protein syndrome. J Clin Lab Anal 13:116-121

23. Wang D, Pascual JM, Iserovich P, Yang H, Ma L, Kuang K, Zuniga FA, Sun RP, Swaroop KM, Fischbarg J, De Vivo DC 2003 Functional studies of threonine 310 mutations in Glut1: T310I is pathogenic, causing Glut1 deficiency. J Biol Chem 278:49015-49021

24. Gould GW, Derechin V, James DE, Tordjman K, Ahern S, Gibbs EM, Lienhard GE, Mueckler M 1989 Insulin-stimulated translocation of the HepG2/erythrocyte-type glucose transporter expressed in 3T3-L1 adipocytes. J Biol Chem 264:2180-2184

25. Brockmann K, Wang D, Korenke CG, von Moers A, Ho YY, Pascual JM, Kuang K, Yang H, Ma L, Kranz-Eble P, Fischbarg J, Hanefeld F, De Vivo DC 2001 Autosomal dominant glut-1 deficiency syndrome and familial epilepsy. Ann Neurol 50:476485

26. Abramson J, Smirnova I, Kasho V, Verner G, Kaback HR, Iwata S 2003 Structure and mechanism of the lactose permease of Escherichia coli. Science 301:610-615

27. Huang Y, Lemieux MJ, Song J, Auer M, Wang DN 2003 Structure and mechanism of the glycerol-3-phosphate transporter from Escherichia coli. Science 301:616-620

28. Shi J, Blundell TL, Mizuguchi K 2001 FUGUE: sequence-structure homology recognition using environment-specific substitution tables and structure-dependent gap penalties. J Mol Biol 310:243-257

29. Gerstein M, Levitt M 1998 Comprehensive assessment of automatic structural alignment against a manual standard, the scop classification of proteins. Protein Sci 7:445-456

30. Karlin A, Akabas MH 1998 Substituted-cysteine accessibility method. Methods Enzymol 293:123-145
31. Mueckler M, Makepeace C 2005 Cysteine-scanning mutagenesis and substituted cysteine accessibility analysis of transmembrane segment 4 of the Glut1 glucose transporter. J Biol Chem 280:39562-39568

32. De Vivo D, Wang D, Pascual J 2007 Disorders of glucose transport. In Rosenberg RN, Prusiner SB, DiMauro S, Barchi RL, Nestler EJ (eds) The Molecular and Genetic Basis of Neurologic and Psychiatric Disease. Philadelphia, PA: ButterworthHeinemann, pp 653-662

33. Klepper J, Voit T 2002 Facilitated glucose transporter protein type 1 (GLUT1) deficiency syndrome: impaired glucose transport into brain - a review. Eur J Pediat 161:295-304

34. Klepper J, Engelbrecht V, Scheffer H, van der Knaap MS, Fiedler A 2007 GLUT1 deficiency with delayed myelination responding to ketogenic diet. Pediatr Neurol 37:130-133

35. Salas-Burgos A, Iserovich P, Zuniga F, Vera JC, Fischbarg J 2004 Predicting the three-dimensional structure of the human facilitative glucose transporter glut 1 by a novel evolutionary homology strategy: insights on the molecular mechanism of substrate migration, and binding sites for glucose and inhibitory molecules. Biophys J 87:2990-2999

36. Doege H, Schurmann A, Ohnimus H, Monser V, Holman GD, Joost HG 1998 Serine-294 and threonine-295 in the exofacial loop domain between helices 7 and 8 of glucose transporters (GLUT) are involved in the conformational alterations during the transport process. Biochem J 329:289-293

37. Cunningham P, Afzal-Ahmed I, Naftalin RJ 2006 Docking studies show that D-glucose and quercetin slide through the transporter GLUT1. J Biol Chem 281:5797-5803

38. Olsowski A, Monden I, Keller K 1998 Cysteine-scanning mutagenesis of flanking regions at the boundary between external loop I or IV and transmembrane segmen II or VII in the GLUT1 glucose transporter. Biochemistry 37:10738-10745

39. Wong HY, Law PY, Ho YY 2007 Disease-associated Glut1 single amino acid substitute mutations S66F, R126C, and T295M constitute Glut1-deficiency states in vitro. Mol Genet Metab 90:193-198

40. Jensen MO, Yin Y, Tajkhorshid E, Schulten K 2007 Sugar transport across lactose permease probed by steered molecular dynamics. Biophys J 93:92-102 